OPEN ACCESS

Edited by:

Hideo Shojaku,

University of Toyama, Japan

Reviewed by:

Fumiyuki Goto

Tokai University Isehara

Hospital, Japan

Toshiaki Yamanaka,

Nara Medical University, Japan

*Correspondence:

Gang Yu

yugangent163@163.com

Specialty section:

This article was submitted to

Neuro-Otology,

a section of the journal

Frontiers in Neurology

Received: 19 July 2019

Accepted: 16 September 2019

Published: 01 October 2019

Citation:

Chen G, Yu G, Li Y, Zhao X, Dai X and Wang G (2019) Cervical Vestibular Evoked Myogenic Potentials in Benign Paroxysmal Positional Vertigo: A Systematic Review and Meta-Analysis. Front. Neurol. 10:1043. doi: 10.3389/fneur.2019.01043

\section{Cervical Vestibular Evoked Myogenic Potentials in Benign Paroxysmal Positional Vertigo: A Systematic Review and Meta-Analysis}

\author{
Gang Chen, Gang Yu*, Yun Li, Xuening Zhao, Xiaoyan Dai and Guotao Wang \\ Department of Otolaryngology Head and Neck Surgery, Shandong Provincial Hospital, Jinan, China
}

Objective: The objective of our study was to investigate the potential association between the occurrence of benign paroxysmal positional vertigo (BPPV) and saccular dysfunction using cervical vestibular evoked myogenic potentials (cVEMP) testing.

Methods: The databases including Pubmed, Embase, and CENTRAL were systemically searched for case-control literatures investigating saccular dysfunction using cVEMP testing in BPPV patients compared with healthy controls. The literatures were published up to 16 April 2019 and were limited to the English language. All statistical processes were carried out using software Review Manager, version 5.3. Subgroup analysis and sensitive analysis were performed simultaneously.

Results: Of the 12 case-control studies confirmed for meta-analysis, p13 latency of cVEMP was assessed in 8 studies, n23 latency in 6 studies, amplitude in 5 studies, asymmetry ratio (AR) in 3 studies, proportion of absent response in 9 studies, and abnormal cVEMP in 8 studies. Compared with healthy controls, the p13 mean latency of cVEMP was longer $(\mathrm{MD}=0.88,95 \% \mathrm{Cl}=0.64-1.12, p<0.00001)$, the mean amplitude was lower (SMD $=-0.60,95 \% \mathrm{Cl}=-0.80$ to $-0.41, p<0.00001)$, and the proportions of absent response $(\mathrm{OR}=8.76,95 \% \mathrm{Cl}=2.28-33.61, p=0.002)$, and abnormal cVEMP (OR $=7.47,95 \% \mathrm{Cl}=4.65-12.01, p<0.00001)$ were higher in BPPV patients. But there was no significant difference in the n23 mean latency $(\mathrm{MD}=0.37,95 \% \mathrm{Cl}=-0.23-0.98, p=0.22)$ and the AR of cVEMP (MD = 3.95, $95 \% \mathrm{Cl}=-4.75-12.65, p=0.37$ ) between BPPV patients and healthy controls. In the sub-group analysis based on age, only the result of the proportion of absent response of cVEMP indicated a significant difference existed $(p=0.002)$ between the studies with age-matched controls $(\mathrm{OR}=2.78,95 \% \mathrm{Cl}=1.09-7.10, p=0.03$ ) and the studies without age-matched controls $(\mathrm{OR}=53.85,95 \% \mathrm{Cl}=10.09-287.13$, $p<0.00001)$. In the sub-group analysis of the proportion of abnormal cVEMP according to the diagnostic criteria of abnormal cVEMP, the result indicated no significant difference existed between the four groups $\left(p=0.61, R^{2}=0 \%\right.$ ). In the sensitivity analysis, we obtained the consistent results after removing each study sequentially. 


\begin{abstract}
Conclusion: The meta-analysis reveals that saccular dysfunction may be associated with BPPV occurrence, and neural degeneration in the saccular macula may be a potential pathogenesis for BPPV.
\end{abstract}

Keywords: benign paroxysmal positional vertigo, cervical vestibular evoked myogenic potentials, saccule, systematic review, meta-analysis

\section{INTRODUCTION}

Vestibular evoked myogenic potentials (VEMPs) are shortlatency and vestibule-dependent electromyographic (EMG) activities evoked by air-conducted loud sound (tone burst or click) (1), bone-conducted vibration (2), or galvanic stimuli (3). Generally, VEMPs contain cervical VEMPs and ocular VEMPs according to different effectors. Cervical VEMPs (cVEMPs), which were first described by Colebatch and Halmagyi (4), are biphasic surface potentials recorded from the sternocleidomastoid (SCM) muscles. They represent inhibition of the ipsilateral vestibulo-collic reflex, and reflect predominantly saccular and inferior vestibular nerve functions (5). Ocular VEMPs (oVEMPs) were found to be optimally recorded below the eyes opposite the stimulated ears, and to originate from the inferior oblique (IO) muscles by Rosengren et al. (6). Although there are still some controversies, oVEMPs may represent activation of the contralateral vestibulo-ocular reflex and reflect predominantly utricular and superior vestibular nerve functions (7). Consequently, VEMPs have become a popular evaluation measure of the otolith function over the past 20 years (8).

Benign paroxysmal positional vertigo (BPPV) is one of the most common peripheral vestibular diseases in specialist clinics of vertigo (9). It is characterized by the presence of brief and episodic vertigo or dizziness in response to head movement relative to gravity. Most BPPV cases having no clear etiology are divided into idiopathic BPPV. The pathogenesis of idiopathic $\mathrm{BPPV}$ is suspected to relate to degenerative process of the saccular or utricular macula (10). Based on the close anatomy relationship, utricular dysfunction is probably regarded as the pathogenesis of BPPV (11). However, the degenerative changes affect both the utricular and saccular maculae because of the anatomical and histological similarities between them (10). Otolith dysfunction derived from degenerative changes may cause the otoconia to detach from the saccular or utricular macula more easily (12).

So far many studies have investigated saccular dysfunction using cVEMP testing in BPPV patients compared with healthy controls, but the results are still inconsistent (10, 13-15). Furthermore, there are several parameters in cVEMP testing, such as latency, amplitude, asymmetry ratio (AR) and so on (16). Different studies used different parameters of cVEMP to analyze, so the conclusions of them were inevitably biased. So we systemically retrieved as many studies as possible and evaluated each parameter of cVEMP testing between BPPV patients and healthy controls. The objective of our study was to investigate the potential association between the BPPV occurrence and saccular dysfunction using cVEMP testing. As far as we know, this is the first systematic review and meta-analysis to investigate the cVEMP results in BPPV patients compared with healthy individuals.

\section{MATERIALS AND METHODS}

\section{Literature Search Strategy}

A literature search was conducted to retrieve all studies which investigated the cVEMP testing between BPPV patients and healthy controls. The databases including Pubmed, Embase, and CENTRAL were systemically searched for all relevant literatures. The literatures were published up to 16 April 2019 and were limited to the English language. The search strategies were "benign paroxysmal positional vertigo" and "vestibular evoked myogenic potential." All the studies were sequentially examined through titles and abstracts screening, and full-text reading to identify studies to meta-analyze. All references of the included literatures were searched additionally. The flowchart is presented in Figure 1.

\section{Study Selection Criteria}

Studies were included in the meta-analysis if they met the inclusion criteria: (1) retrospective or prospective case-control studies about cVEMP testing comparing BPPV patients with healthy controls; (2) diagnosis of BPPV relied on brief and recurrent vertigo and characteristic nystagmus in positional tests, such as Dix-Hallpike test and supine Roll test; (3) cVEMP outcomes in BPPV and healthy groups, such as latency, amplitude, AR, proportion of absent cVEMP, or proportion of abnormal cVEMP, were clearly stated in whole or in part; (4) healthy controls were subjects with normal hearing and no known history of vestibular and neurological disorders. If the data of literatures were duplicated, we selected the articles with the latest publication and complete data. The exclusion criteria were as follows: (1) case reports, reviews, comments, letters or practice guidelines; (2) absence of healthy controls; (3) unclear diagnosis of BPPV; (4) insufficient data of cVEMP to extract to compare BPPV patients with healthy controls; (5) BPPV patents with conductive hearing loss, or neurological diseases, or other otologic diseases (vestibular neuronitis, Meniere's disease, sudden sensorineural hearing loss, chronic otitis media, labyrinthitis, ototoxicity).

\section{Data Extraction}

Data extraction and quantification were independently performed by two authors (GC and GY). Agreement on all the details was reached by discussion or by appealing to a third author. The following data were extracted from each study: first author, year of publication, country, research type, number of BPPV patients or healthy controls included, age, gender, 


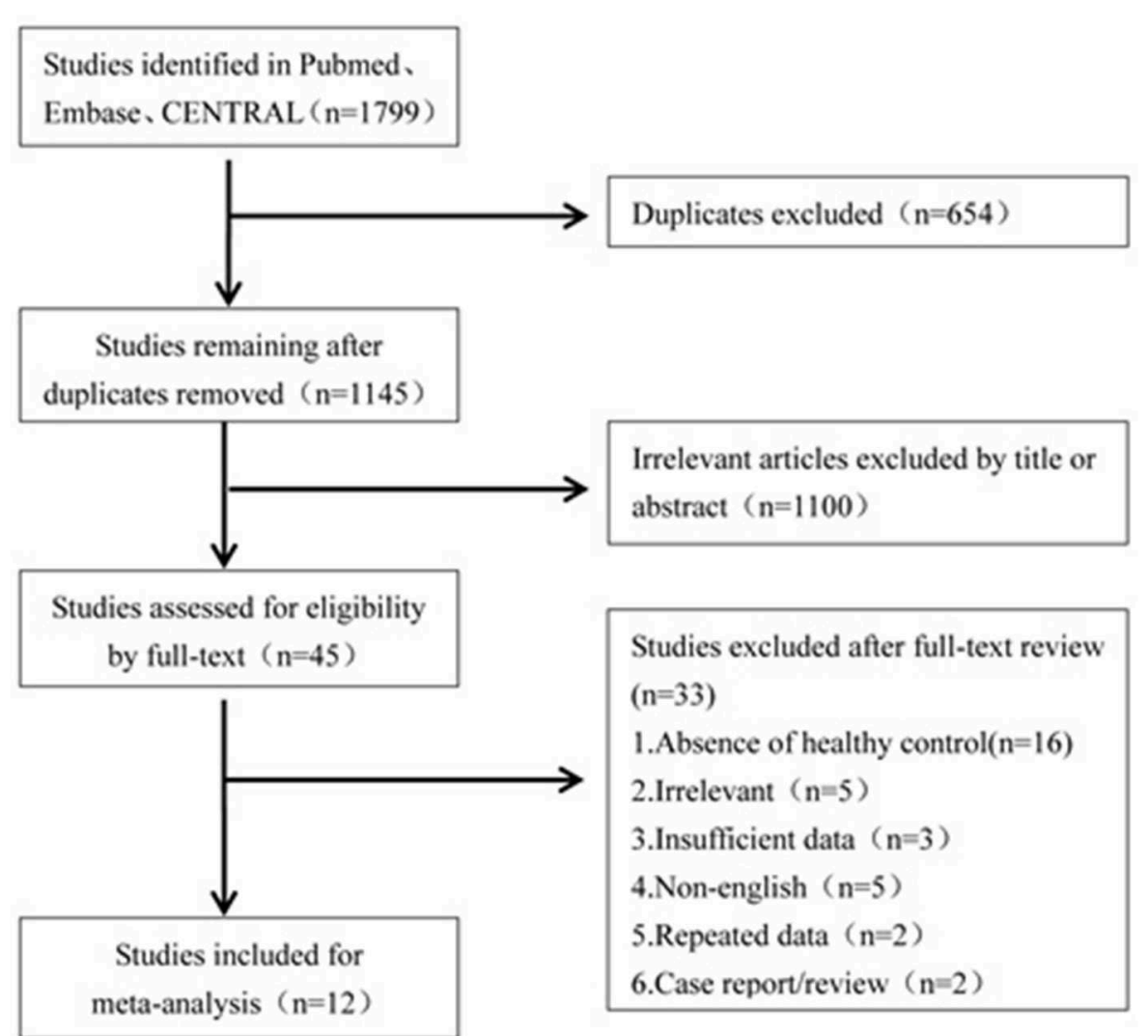

FIGURE 1 | Flowchart of data search and studies selection for meta-analysis.

number of each type of BPPV, number of included ears, type of acoustic stimuli, data of each parameter of cVEMP (p13 latency, n23 latency, p13-n23 peak to peak amplitudes, AR, number of ears with absent response, criteria for abnormal cVEMP, and number of ears with abnormal cVEMP). AR was calculated as $100[(\mathrm{Au}-\mathrm{Aa}) /(\mathrm{Au}+\mathrm{Aa})]$, where $\mathrm{Au}$ is the p13-n23 amplitude on the unaffected side and Aa is the p13-n23 amplitude on the affected side.

\section{Quality Assessment}

Two authors (GC and GY) performed the quality assessment according to the Newcastle-Ottawa Scale (NOS). Disagreement was resolved by appealing to a third author. Study quality was considered as high when the score had 6 or more stars (17).

\section{Statistical Analysis}

All statistical processes were carried out using software Review Manager (RevMan), version 5.3. Mean difference (MD) and its $95 \%$ confidence interval (CI) were calculated to analyze p13 latency, n23 latency and AR, standardized mean difference (SMD) and its 95\% CI were used to analyze peak to peak amplitude, and odds ratios (OR) and its 95\% CI were used to analyze dichotomous variables between two groups. We used $\mathrm{X} 2$ and $\mathrm{I}^{2}$ index to evaluate the statistical heterogeneity in the meta-analysis. If $p<0.05$ or $I^{2}>50 \%$, the random-effects model was selected because of the significant heterogeneity, otherwise the fixed-effects model was selected. Considering the age may affect the results of cVEMP, sub-group analysis was performed based on whether or not the age matched between BPPV and healthy groups (18). So far, there has been no uniform diagnostic criterion for abnormal cVEMP. Then we performed a sub-group analysis according to the different diagnostic criteria of abnormal cVEMP. Publication bias of our meta-analysis was evaluated through funnel plots.

\section{RESULTS}

\section{Literature Screening}

Of the 1,799 potentially relevant literatures, 654 studies were firstly removed for duplication. After screening titles and abstracts, 1,100 articles were excluded for irrelevance to our purpose. Of the remaining, 45 articles needed a full text screening, 16 were excluded for lack of healthy controls, 5 were excluded for irrelevance to our purpose, 5 were excluded for nonEnglish publication, 2 were excluded for case report or reviews, and 2 were excluded because they came from the same team and the data may have an overlap with the third article. The third article with latest publication and complete data was then included (19). Finally, we confirmed 12 articles for meta-analysis $(10,13-15,19-26)$. 


\section{Characteristics of Studies Included}

Twelve articles involved 537 BPPV patients and 489 healthy controls. Because bilateral BPPV cases were included in two studies $(10,14)$ and both ears of healthy controls in most studies were tested together, 545 ears in BPPV groups and 871 ears in healthy controls were included for cVEMP analysis. Of the 12 studies, 6 were prospective case-control studies $(10,15,20,21$, $25,26)$, and 8 included age-matched healthy controls $(10,13$, 14, 19, 21-24). Eleven studies definitely excluded the individuals with conductive hearing loss in BPPV and control groups, except the study of Martínez Pascual et al. (20). cVEMP testing in all 12 studies were conducted through air-conducted sound. The characteristics of 12 included articles are described in Table $\mathbf{1 .}$ The detailed results of cVEMP testing for BPPV patients and healthy controls are shown in Table 2.

\section{Quality Assessment}

We used NOS to assess the quality of the 12 eligible studies. The NOS scores ranged from 6 to 7 stars, and were all highquality studies, as shown in Table 3. The main deduction items were the representativeness of the cases, selection of controls, and non-response rate.

\section{Meta-Analysis Results} P13 Latency of cVEMP in BPPV and Healthy Groups Eight studies $(10,13-15,19,22,25,26)$ compared mean p13 latency of cVEMP in BPPV and healthy groups. Because of no significant heterogeneity ( $p=0.07, I^{2}=46 \%$, Figure 2 ), fixedeffects model was selected. The mean p13 latency of cVEMP in BPPV patients was significantly longer than that in healthy controls according to the forest plot $(\mathrm{MD}=0.88,95 \% \mathrm{CI}=0.64-$ $1.12, p<0.00001$, Figure 2). In the sub-group analysis, no significant difference existed ( $p=0.14$, Figure 2 ) between the five studies with age-matched controls $(\mathrm{MD}=0.77,95 \% \mathrm{CI}=0.49$ $1.05, p<0.00001$, Figure 2) $(10,13,14,19,22)$ and the three studies without age-matched controls $(\mathrm{MD}=1.18,95 \%$ $\mathrm{CI}=0.71-1.64, p<0.00001$, Figure 2$)(15,25,26)$.

\section{N23 Latency of cVEMP in BPPV and Healthy Groups}

Six studies $(10,14,15,19,22,25)$ compared mean n23 latency of cVEMP in BPPV and healthy groups. Because of a significant heterogeneity ( $p=0.009, I^{2}=67 \%$, Figure 3 ), random-effects model was selected. The mean n23 latency of cVEMP in BPPV patients was not different from that in healthy controls according to the forest plot $(\mathrm{MD}=0.37,95 \% \mathrm{CI}=-0.23-0.98, p=0.22$, Figure 3). In the sub-group analysis, no significant difference

TABLE 1 | The basic characteristics of all eligible studies.

\begin{tabular}{|c|c|c|c|c|c|c|c|c|c|c|c|c|}
\hline References & Country & $\begin{array}{l}\text { Study } \\
\text { type }\end{array}$ & Groups & No. & Gender(M:F) & $\begin{array}{l}\text { Age(years) } \\
\text { (mean } \pm \text { SD) }\end{array}$ & $\begin{array}{c}\text { Age } \\
\text { matched }\end{array}$ & $\begin{array}{l}\text { PSC- } \\
\text { BPPV }\end{array}$ & $\begin{array}{l}\text { HSC- } \\
\text { BPPV }\end{array}$ & $\begin{array}{l}\text { ASC- } \\
\text { BPPV }\end{array}$ & $\begin{array}{c}\text { Bilateral } \\
\text { BPPV }\end{array}$ & $\begin{array}{l}\text { MSC- } \\
\text { BPPV }\end{array}$ \\
\hline \multirow[t]{2}{*}{ Akkuzu et al. (10) } & Turkey & Prospective & BPPV & 25 & $6: 19$ & $52.9 \pm 11.9$ & Yes & 25 & 0 & 0 & 5 & 0 \\
\hline & & & Control & 17 & $6: 11$ & $51.8 \pm 15.8$ & & - & - & - & - & - \\
\hline \multirow[t]{2}{*}{ Yang et al. (15) } & Korea & Prospective & BPPV & 41 & $12: 29$ & Mean 59 & No & 34 & 7 & 0 & 0 & 0 \\
\hline & & & Control & 92 & NA & Mean 42 & & - & - & - & - & - \\
\hline \multirow[t]{2}{*}{ Korres et al. (14) } & Greece & NA & BPPV & 27 & $14: 13$ & Median 45 & Yes & 27 & 0 & 0 & 3 & 0 \\
\hline & & & Control & 30 & $17: 13$ & Median 47 & & - & - & - & - & - \\
\hline \multirow[t]{2}{*}{ Longo et al. (25) } & Italy & Prospective & BPPV & 23 & $8: 15$ & Mean 59 & No & 23 & 0 & 0 & 0 & 0 \\
\hline & & & Control & 24 & $12: 12$ & Mean 51 & & - & - & - & - & - \\
\hline \multirow[t]{2}{*}{ Eryaman et al. (26) } & Turkey & Prospective & BPPV & 31 & $12: 19$ & $51.9 \pm 11.8$ & No & 31 & 0 & 0 & 0 & 0 \\
\hline & & & Control & 23 & $8: 15$ & $51.1 \pm 10.8$ & & - & - & - & - & - \\
\hline \multirow{2}{*}{$\begin{array}{l}\text { Nakahara et al. } \\
(24)\end{array}$} & Japan & NA & BPPV & 12 & $5: 7$ & Mean 65.5 & Yes & 12 & 0 & 0 & 0 & 0 \\
\hline & & & Control & 12 & $6: 6$ & Mean 63.1 & & - & - & - & - & - \\
\hline \multirow[t]{2}{*}{ Talaat et al. (23) } & Egypt & NA & BPPV & 112 & $52: 60$ & $46.2 \pm 10.2$ & Yes & 112 & 0 & 0 & 0 & 0 \\
\hline & & & Control & 100 & $45: 55$ & $44.2 \pm 9.9$ & & - & - & - & - & - \\
\hline \multirow[t]{2}{*}{ Singh et al. (19) } & India & NA & BPPV & 31 & NA & $42 \pm 5.7$ & Yes & 31 & 0 & 0 & 0 & 0 \\
\hline & & & Control & 31 & NA & $42.2 \pm 5.8$ & & - & - & - & - & - \\
\hline \multirow[t]{2}{*}{ Kim et al. (13) } & Korea & NA & BPPV & $102^{*}$ & $48: 54$ & $62.8 \pm 13.1$ & Yes & 47 & 51 & 0 & 0 & 4 \\
\hline & & & Control & 50 & $23: 27$ & $60.1 \pm 9.2$ & & - & - & - & - & - \\
\hline \multirow[t]{2}{*}{ Karatas et al. (22) } & Turkey & NA & BPPV & 36 & $10: 26$ & Mean 47.2 & Yes & 34 & 2 & 0 & 0 & 0 \\
\hline & & & Control & 20 & $7: 13$ & Mean 45.1 & & - & - & - & - & - \\
\hline \multirow[t]{2}{*}{ Xu et al. (21) } & China & prospective & BPPV & 30 & $12: 18$ & Mean 45.5 & Yes & 30 & 0 & 0 & 0 & 0 \\
\hline & & & Control & 30 & $10: 20$ & Mean 42.2 & & - & - & - & - & - \\
\hline \multirow[t]{2}{*}{ Pascual et al. (20) } & Spain & prospective & BPPV & 67 & $16: 51$ & Mean 58.06 & No & 67 & 0 & 0 & 0 & 0 \\
\hline & & & Control & 60 & $23: 37$ & Mean 46.3 & & - & - & - & - & - \\
\hline
\end{tabular}

*Ten patients with bilateral BPPV were excluded.

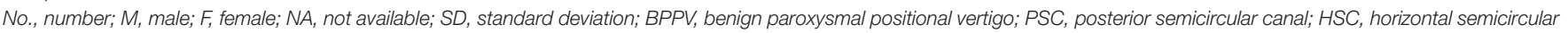
canal; ASC, anterior semicircular canal; MSC, multiple semicircular canal. 
TABLE 2 | The detailed results of cervical vestibular evoked myogenic potentials (cVEMP) test.

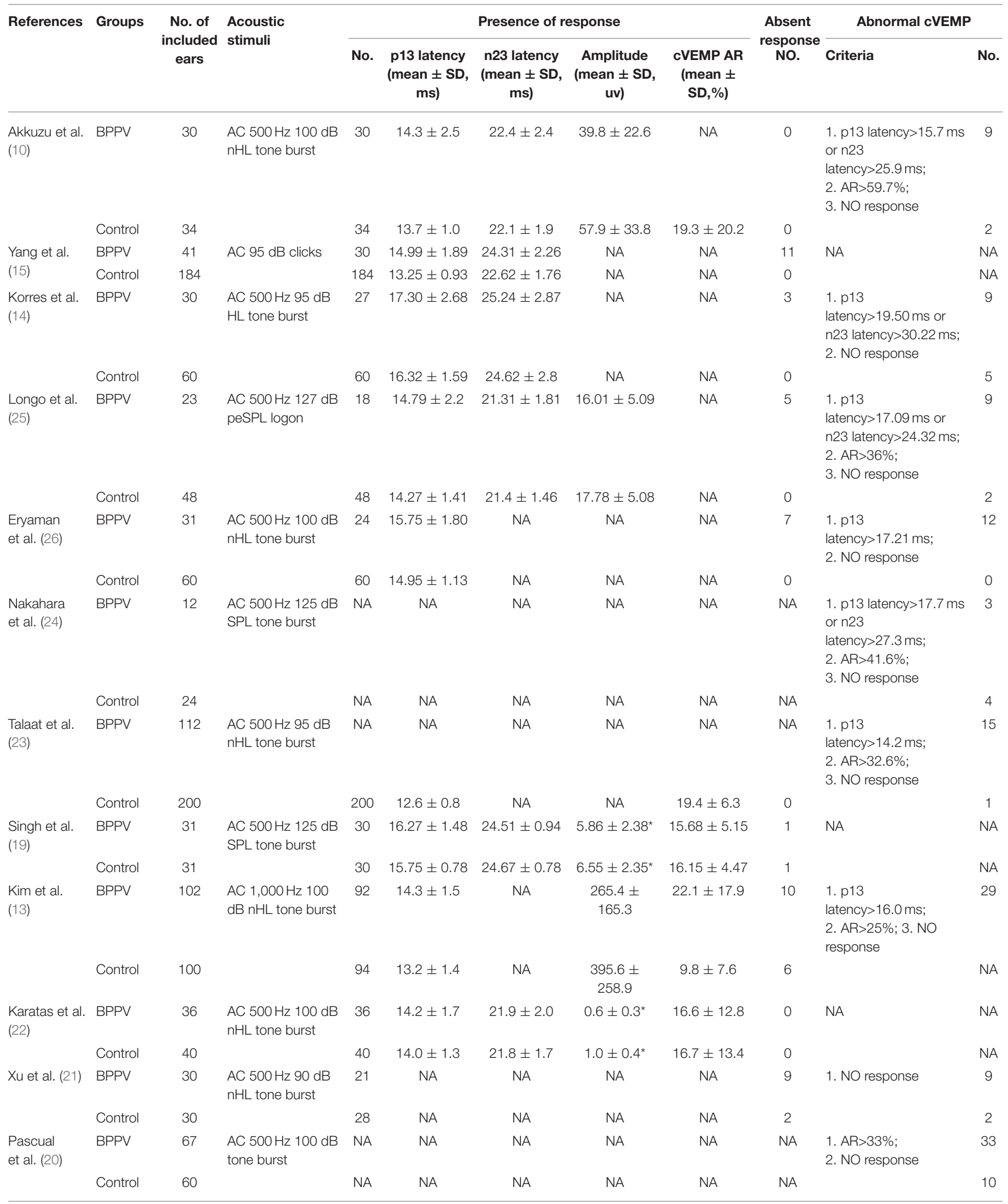

${ }^{*}$ CVEMP amplitudes were corrected using the background electromyographic activities of the sternocleidomastoid.

BPPV, benign paroxysmal positional vertigo; cVEMP, cervical vestibular evoked myogenic potentials; NA, not available; SD, standard deviation; No., number; AR, asymmetry ratio; AC, air-conducted; HL, hearing level; $\mathrm{nHL}$, normal hearing level; SPL, sound pressure level; peSPL, peak equivalent sound pressure level. 
existed ( $p=0.37$, Figure 3) between the four studies with age-matched controls $(\mathrm{MD}=-0.01,95 \% \mathrm{CI}=-0.36-0.34$, $p=0.97$, Figure 3) $(10,14,19,22)$ and two studies without age-matched controls $(\mathrm{MD}=0.81,95 \% \mathrm{CI}=-0.93-2.56$, Figure 3) (15, 25).

\section{Amplitude of cVEMP in BPPV and Healthy Groups}

Five studies $(10,13,19,22,25)$ assessed the peak to peak amplitude of cVEMP in BPPV and healthy groups. Because of no

TABLE 3 | Quality assessment of the selected studies using the Newcastle-Ottawa Scale (NOS).

\begin{tabular}{llccc}
\hline References & \multicolumn{3}{c}{ Score on dimensions } & \multirow{2}{*}{ Score } \\
\cline { 2 - 4 } & Selection & Comparability & Exposure & \\
\hline Akkuzu et al. (10) & 3 & 2 & 2 & 7 \\
Yang et al. (15) & 3 & 1 & 2 & 6 \\
Korres et al. (14) & 2 & 2 & 2 & 6 \\
Longo et al. (25) & 3 & 1 & 2 & 6 \\
Eryaman et al. (26) & 3 & 1 & 2 & 6 \\
Nakahara et al. (24) & 2 & 2 & 2 & 6 \\
Talaat et al. (23) & 3 & 2 & 2 & 7 \\
Singh et al. (19) & 2 & 2 & 2 & 6 \\
Kim et al. (13) & 3 & 2 & 2 & 7 \\
Karatas et al. (22) & 3 & 1 & 2 & 7 \\
Xu et al. (21) & 3 & 2 & 6 \\
Pascual et al. (20) & 3 & & 2 & 7
\end{tabular}

significant heterogeneity ( $p=0.16, I^{2}=39 \%$, Figure 4), fixedeffects model was selected. The mean amplitude of cVEMP in BPPV patients was lower than that in healthy controls according to the forest plot (SMD $=-0.60,95 \% \mathrm{CI}=-0.80$ to $-0.41, p<$ 0.00001 , Figure 4). In the sub-group analysis, the result indicated that no significant difference existed ( $p=0.32$, Figure 4 ) between the four studies with age-matched controls (SMD $=-0.64,95 \%$ $\mathrm{CI}=-0.85$ to $-0.44, p<0.00001$, Figure 4$)(10,13,19,22)$ and one study without age-matched controls (SMD $=-0.34,95 \%$ $\mathrm{CI}=-0.89-0.20, p=0.22$, Figure 4) (25).

\section{AR of cVEMP in BPPV and Healthy Groups}

Three studies $(13,19,22)$ assessed the AR of cVEMP in BPPV and healthy groups. Because of a significant heterogeneity $(p<$ $0.00001, I^{2}=93 \%$, Figure 5), random-effects model was selected. The AR of cVEMP in BPPV patients was not significantly different from that in healthy controls according to the forest plot $(\mathrm{MD}=3.95,95 \% \mathrm{CI}=-4.75-12.65, p=0.37$, Figure 5). Considering all the three studies with age-matched control, no sub-group analysis was performed.

\section{Proportion of Absent Response of cVEMP in BPPV and Healthy Groups}

Nine studies (10,13-15, 19, 21, 22, 25, 26) assessed the proportion of absent response of cVEMP in BPPV and healthy groups. Because of a significant heterogeneity $\left(p=0.02, I^{2}=61 \%\right.$, Figure 6), random-effects model was selected. The proportion of absent response of cVEMP in BPPV patients was higher than that in healthy controls according to the forest plot $(\mathrm{OR}=8.76$, $95 \%$ CI $=2.28-33.61, p=0.002$, Figure 6). In the sub-group analysis, the result indicated a significant difference existed

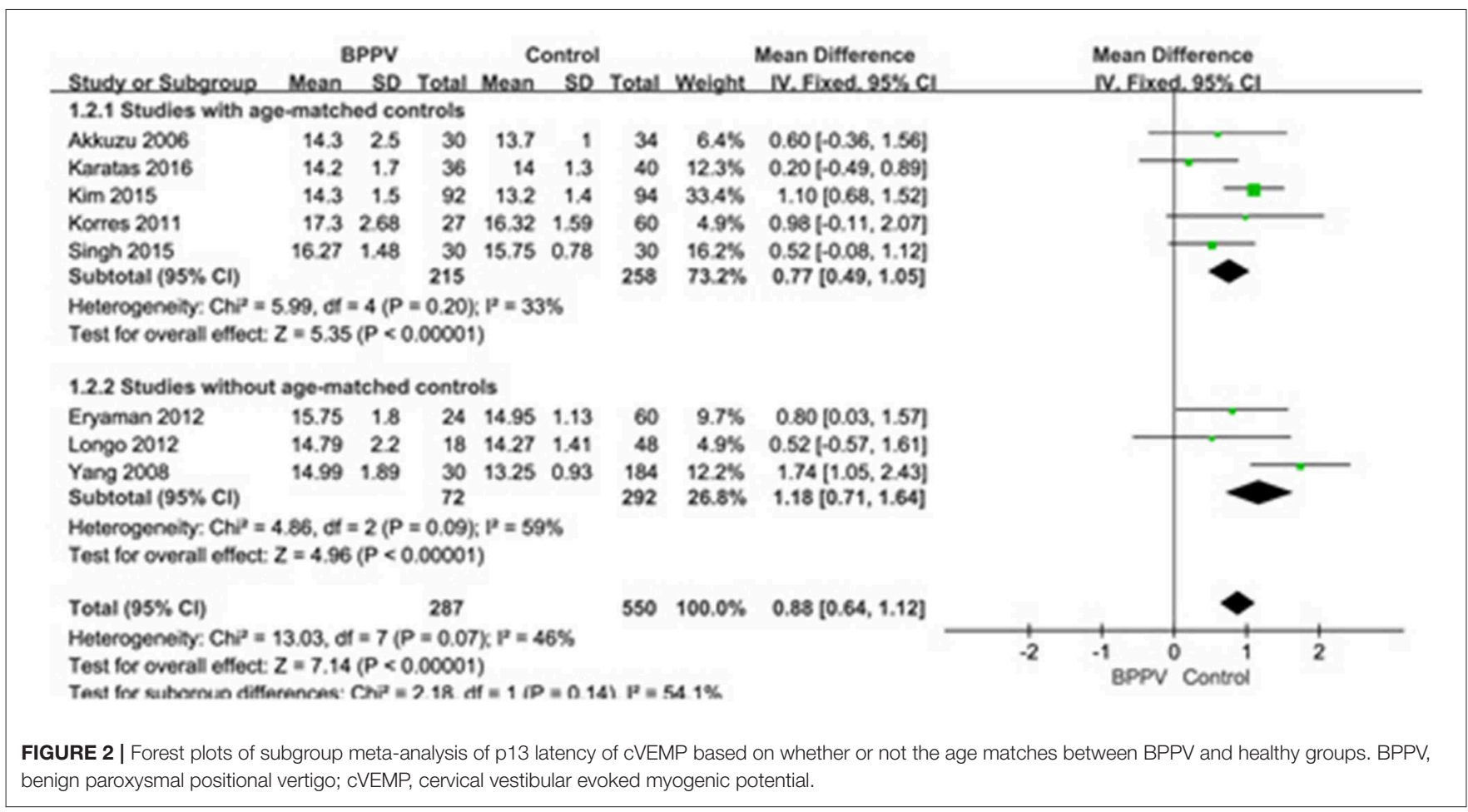




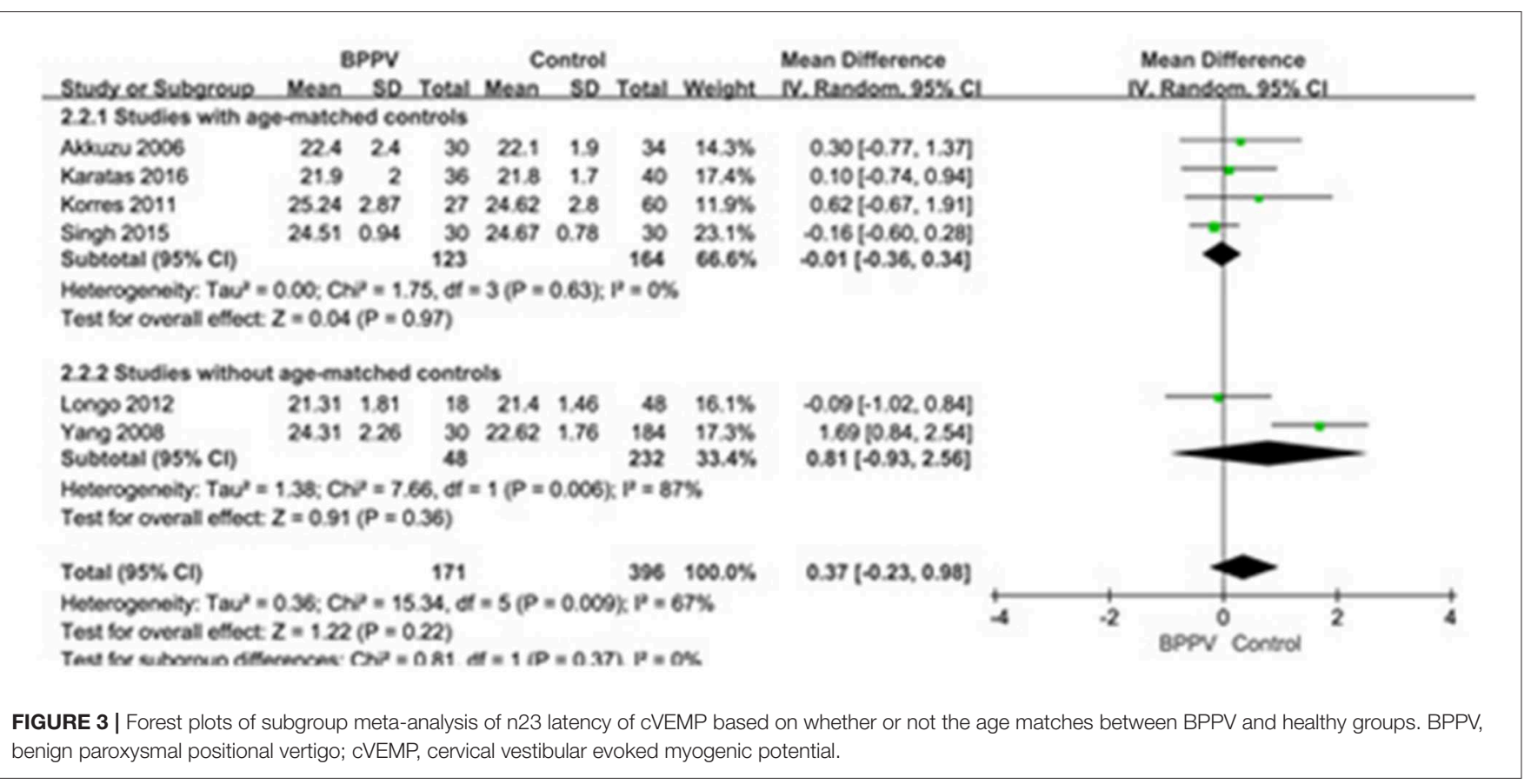

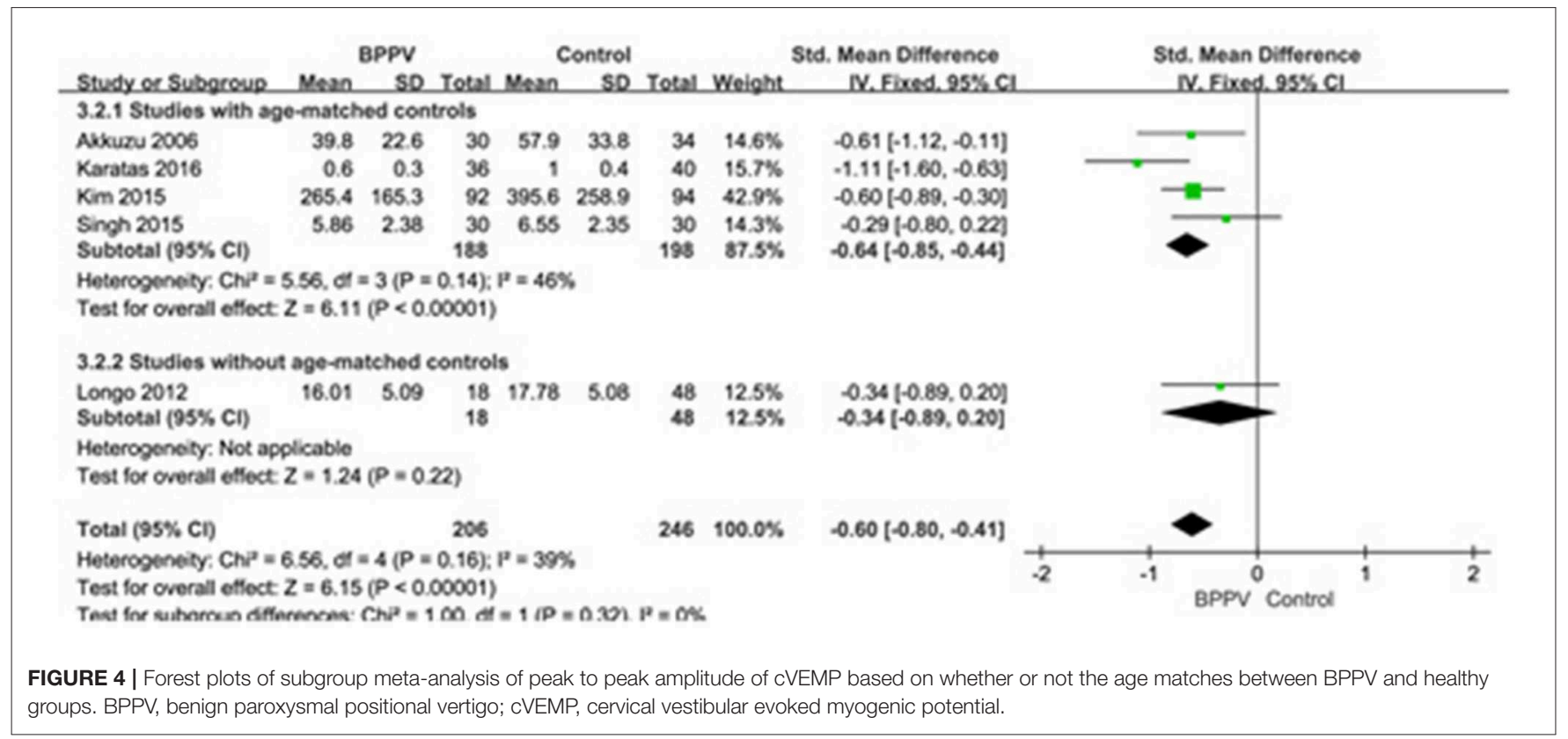

( $p=0.002$, Figure 6) between the six studies with age-matched controls $(\mathrm{OR}=2.78,95 \% \mathrm{CI}=1.09-7.10, p=0.03$, Figure 6) $(10,13,14,19,21,22)$ and three studies without age-matched controls $(\mathrm{OR}=53.85,95 \% \mathrm{CI}=10.09-287.13, p<0.00001$, Figure 6) $(15,25,26)$.

\section{Proportion of Abnormal cVEMP in BPPV and Healthy Groups}

Eight studies $(10,14,20,21,23-26)$ assessed the proportion of abnormal cVEMP in BPPV and healthy groups. Because of no significant heterogeneity ( $p=0.20, I^{2}=28 \%$, Figure 7 ), fixed-effects model was selected. The proportion of abnormal cVEMP in BPPV patients was higher than that in healthy controls according to the forest plot (OR $=7.47,95 \%$ $\mathrm{CI}=4.65-12.01, p<0.00001$, Figure 7). In the sub-group analysis based on age, the result indicated no significant difference existed ( $p=0.61$, Figure 7 ) between the five studies with age-matched controls $(\mathrm{OR}=7.47,95 \% \mathrm{CI}=4.65-$ 12.01, $p<0.00001$, Figure 7) $(10,14,21,23,24)$ and three studies without age-matched controls ( $O R=8.49,95 \%$ 


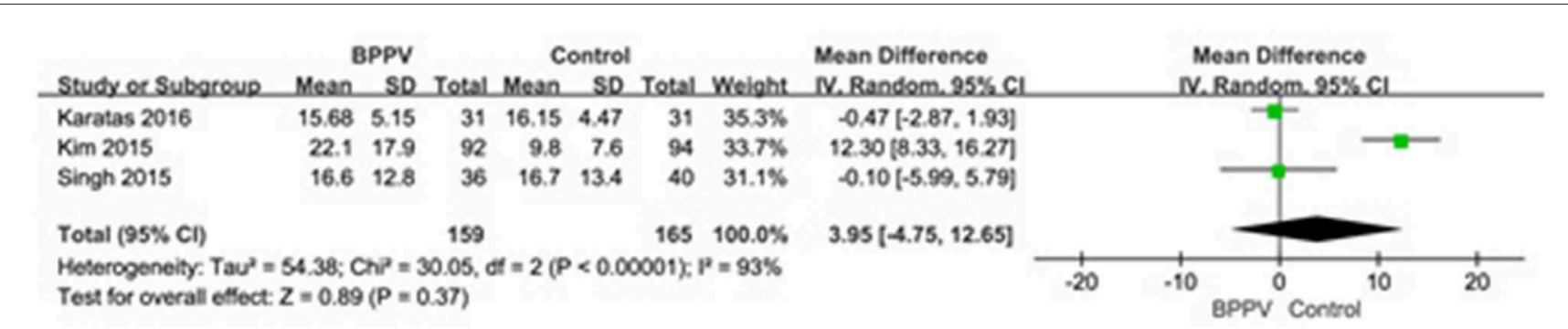

FIGURE 5 | Forest plots of meta-analysis of asymmetry ratio (AR) of cVEMP between BPPV and healthy groups. BPPV, benign paroxysmal positional vertigo; cVEMP, cervical vestibular evoked myogenic potential.

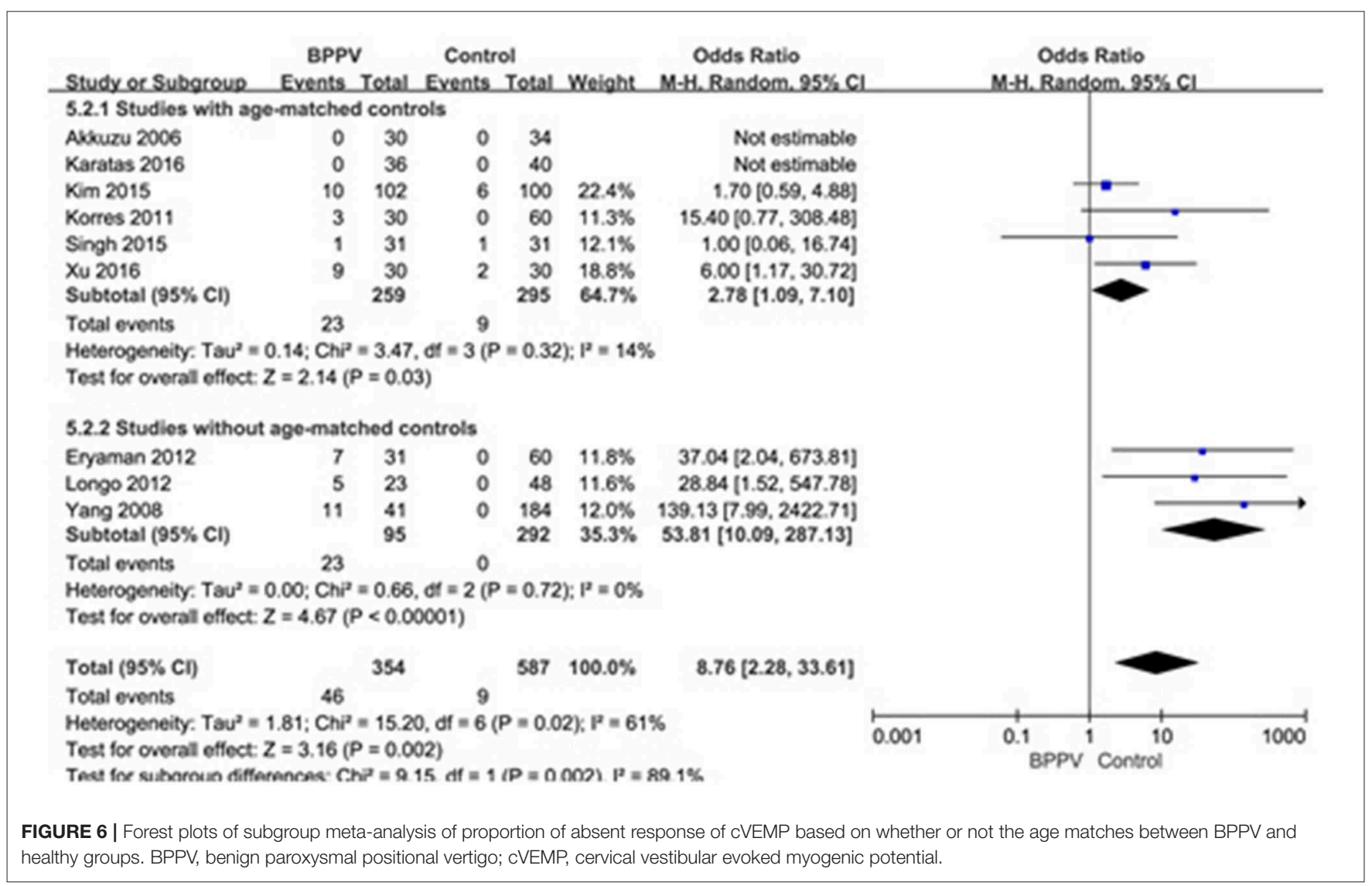

$\mathrm{CI}=4.29-16.80, p<0.00001$, Figure 7$)(20,25,26)$. In the sub-group analysis according to the diagnostic criteria of abnormal cVEMP, the result indicated no significant difference existed between the four groups $\left(p=0.61, I^{2}=0 \%\right.$, Figure 8).

\section{Sensitivity Assessment and Publication Bias}

A sensitivity analysis was performed in each meta-analysis. After removing each study sequentially to evaluate the reliability of our conclusions, we obtained the consistent results (Supplementary Figures). However, after removal of the study of Yang et al. (15), the heterogeneity became insignificant in the meta-analysis of n23 latency ( $p=0.78, I^{2}=0 \%$, Figure 9A). After removal of the study of Kim et al. (13), the heterogeneity became insignificant in the meta-analysis of $\mathrm{AR}(p=0.91$, $I^{2}=0 \%$, Figure 9B). After removal of the studies of Yang et al. (15) and Kim et al. (13), the heterogeneity became insignificant in the meta-analysis of the proportion of absent response of cVEMP ( $p=0.37, I^{2}=6 \%$, Figure 9C). These suggested that the source of high heterogeneity in the three meta-analyses above were from study of Yang et al. (15) or/and the study of Kim et al. (13). Asymmetry was observed in the pool of data from the included literatures, and publication bias was obviously indicated, as shown by funnel plots (Figure 10). 


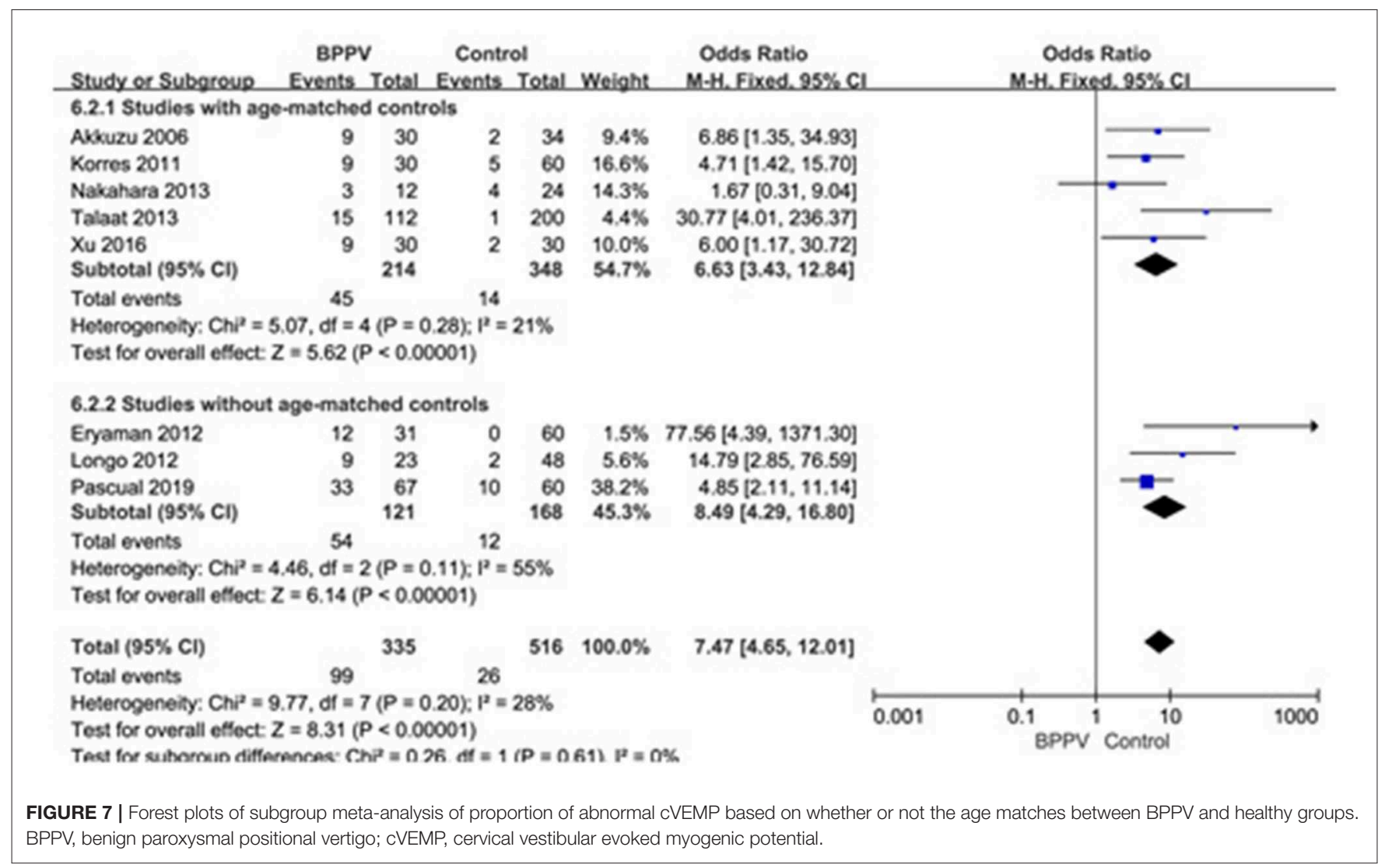

\section{DISCUSSION}

The pathophysiology of BPPV remains unclear, and it is suspected that otolith debris detaches from macula of the otolith membrane, and migrates into the endolymphatic lumen of semicircular canals (27) or attaches to the cupula of the semicircular canals (28). The cause of dislodged otoconia in secondary BPPV may be attributed to head trauma (29) or inner ear diseases such as sudden sensorineural hearing loss (30) and Meniere's disease (31). But otolithic degenerative changes related to age (32) and osteoporosis (33) and so on, may be responsible for the occurrence of idiopathic BPPV. Due to the similarities in anatomy and histology, the degenerative progress not only affects the utricular macula, but also injures saccular macula which can be detected by cVEMP.

Considerable previous studies have reported that there were higher incidences of abnormal cVEMP in the BPPV groups compared with the healthy controls $(13,15,26)$. But so far there has been a controversy about this argument (14, 22, 24). Akkuzu et al. (10) and Korres et al. (14) found that the mean latency of p13 and n23 of cVEMP in BPPV cases was not significantly different from that in healthy individuals. Nakahara et al. (24) reported that the proportion of abnormal cVEMP indicated no significant difference between the two groups. As far as we know, this is the first systematic review and meta-analysis to investigate the cVEMP results in BPPV patients compared with healthy individuals based on 12 case-control studies with high-quality, and to explore possible association between BPPV occurrence and saccular dysfunction.

In our meta-analysis, we first confirmed that the mean p13 latency was significantly longer in the affected ears of BPPV groups than that in healthy controls, but the difference in mean n23 latency between the two groups was statistically insignificant. Gacek et al. (34) performed post-mortem examinations of temporal bones from five BPPV patients and found the loss of vestibular ganglion cells in the inferior vestibular nerve, and neuron degeneration in the saccular nerve which was a part of inferior vestibular nerve. The delay of p13 latency may be associated with the neural degeneration of the saccular nerve. The mechanism has not been understood clearly. We speculate that the disorder of myelin sheath of the saccular nerve which was induced by the degeneration process may be responsible for the delay of p13 latency through reducing nerve conduction velocity $(35,36)$. Besides, prolonged latency of p13 or n23 might indicate the injury of the cVEMP reflex pathway. Retrolabyrinthine lesions, including large cerebellopontine angle tumors, or central disorders including multiple sclerosis, were also responsible for the prolongation of p13 latency mainly due to the vestibulospinal tract lesions (37). Considering that age may affect the latency of p13 and n23 of cVEMP, we performed a sub-group analysis, but the differences were insignificant according to our results $(p=0.14$ for $p 13$ latency and 


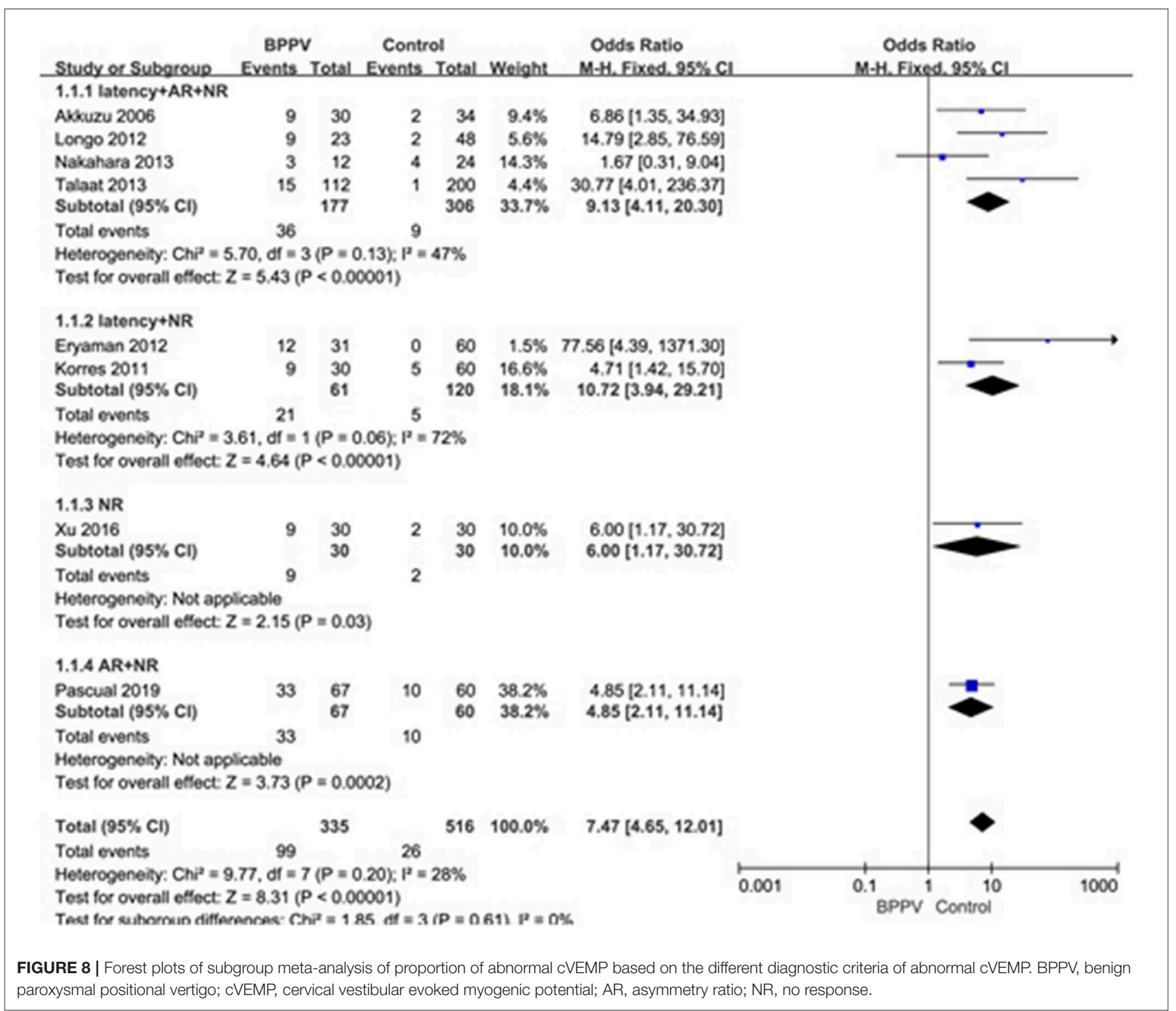

$p=0.37$ for $\mathrm{n} 23$ latency). Therefore, regardless of the age, p13 latency was delayed in BPPV patients but n23 latency was not affected.

Secondly, our analysis also confirmed that the mean amplitude in the affected ears of BPPV groups was significantly lower than that in healthy controls. Because of the low reproducibility and large variation of amplitude of cVEMP, only five studies assessed the difference between the two groups. Two of the studies corrected the amplitude using the background EMG activity $(19,22)$, then we used SMD and its $95 \%$ CI to meta-analyse amplitude, and found that the difference of mean amplitude between BPPV groups and healthy controls was significant with no significant heterogeneity. Considering the age may affect the amplitude of cVEMP, we also performed a sub-group analysis, but the differences were insignificant according our results $(p=0.32)$. This may be due to only one study without age-matched controls being included. In addition, the difference in $\mathrm{AR}$ of cVEMP between the two groups was statistically insignificant, but only three studies were enrolled to meta-analyse with a significant heterogeneity. Kataras et al. (22) found that the amplitudes from the affected and unaffected ears of unilateral BPPV patients were similar, suggesting bilateral involvement of neural degeneration in unilateral BPPV. This may partly explain the insignificant difference of AR in BPPV groups compared with healthy controls.

Thirdly, we also found that the proportion of absent response of cVEMP in the affected ears in BPPV groups was significantly higher than that in healthy controls. The proportion of absent response of cVEMP in the affected ears of BPPV groups ranged from 0 to $30 \%(10,21,22)$ in included studies, resulting in a significant heterogeneity. According to sensitivity analysis, we found the heterogeneity was from the studies of Kim et al. (13) 


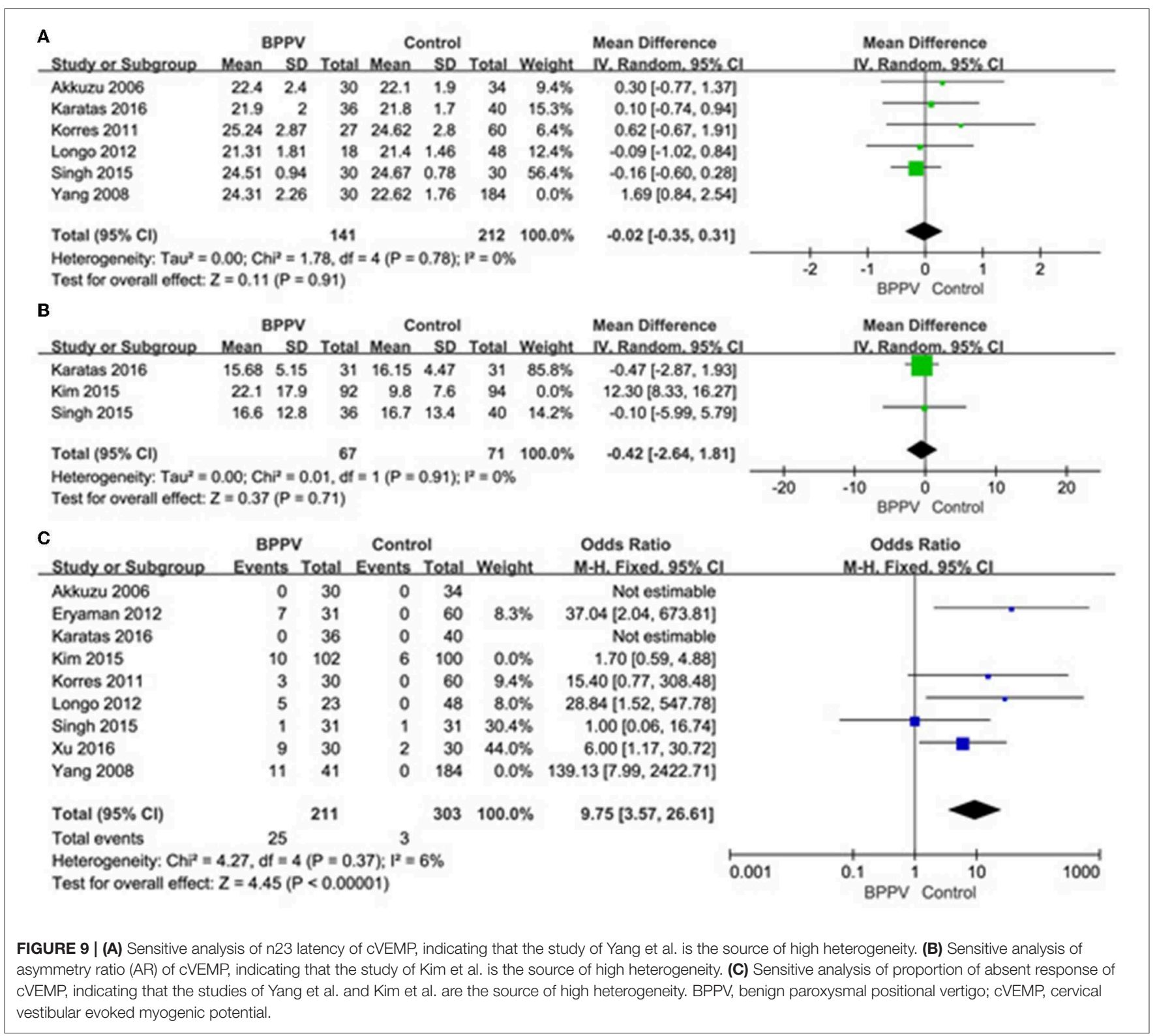

and Yang et al. (15). In the study of Yang et al. (15), up to $26.8 \%$ of affected ears in BPPV groups showed no response in cVEMP while both ears of all individuals in healthy controls showed a response in cVEMP. In the study of Kim et al. (13), the sample size was large and only $9.8 \%$ of affected ears in BPPV groups showed no response of cVEMP. The occurrence of absent response usually indicated that the degeneration of saccular macula was extensive (26). In addition, conductive hearing loss may cause the absence of cVEMP response. Only the study of Martínez Pascual et al. (20) in our included articles did not definitely emphasize the exclusion of conductive hearing loss, but it was not the source of heterogeneity according to sensitivity analysis. Considering the age may affect the proportion of absent response, we performed a sub-group analysis, and the differences were significant according to our results $(p=0.002)$ with high heterogeneity $\left(I^{2}=89.1 \%\right)$. So age might be an important factor for analysis of proportion of absent response of cVEMP.

Lastly, we meta-analyzed the proportion of abnormal cVEMP based on their own criteria for abnormal cVEMP in eight studies included, and found that the proportion of abnormal cVEMP in the affected ears of BPPV groups was significantly higher than that in healthy controls. We performed a sub-group analysis based on age, and the differences were insignificant between studies with and without age-matched controls $(p=0.61)$. The proportion of abnormal cVEMP ranged from 13.4 to $49.2 \%$ $(20,23)$ in our studies included in meta-analysis, which varied rather largely. This was mainly due to diverse criteria for defining abnormality of cVEMP. Then we performed sub-group analysis according to the diagnostic criteria of abnormal cVEMP, and the result indicated no significant difference existed between the four 


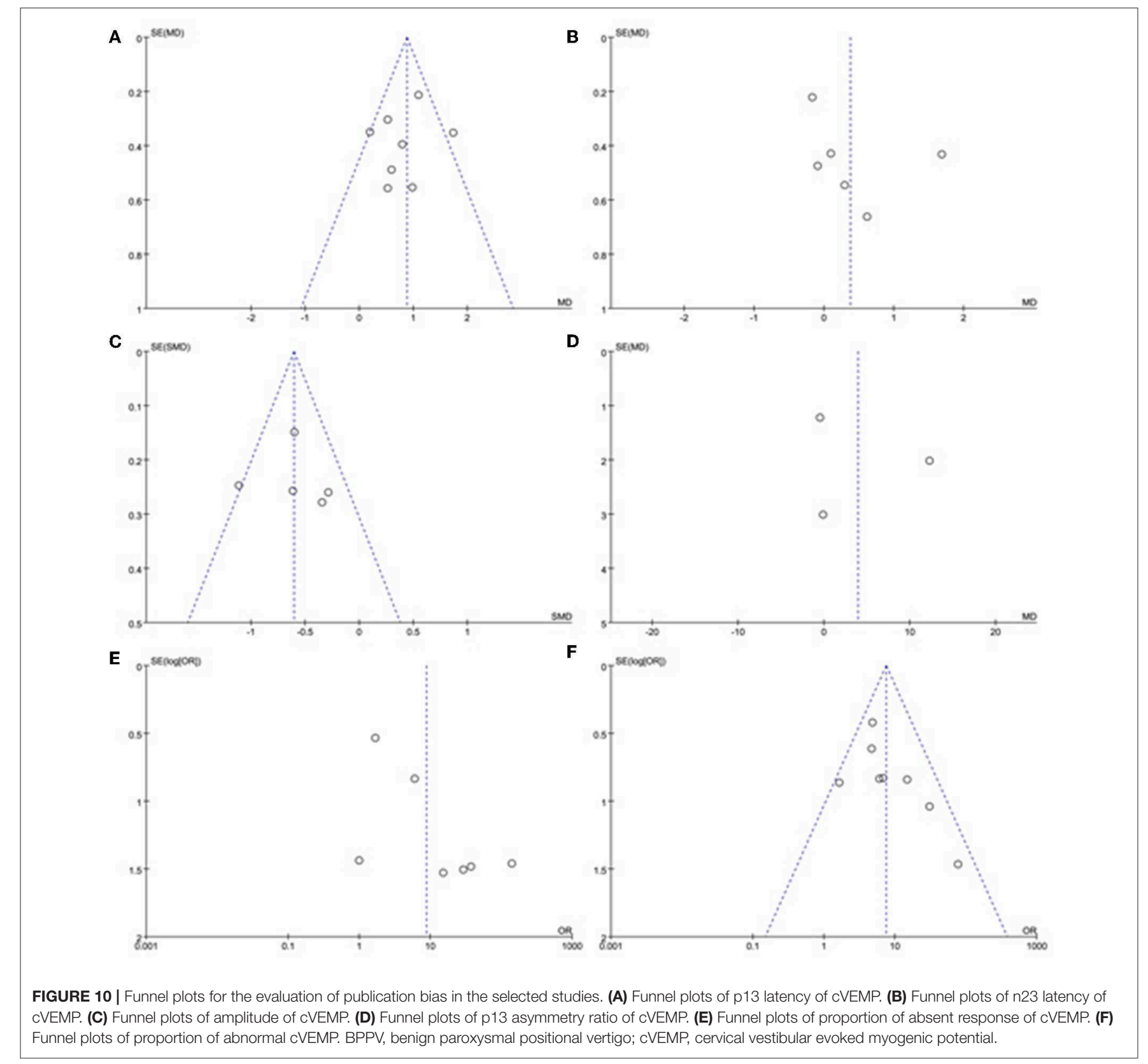

groups. Even so, we should constitute uniform criteria as soon as possible and conduct further studies adopting uniform criteria.

There are still a few limitations to be considered in our meta-analysis. First of all, the sample size of our meta-analysis was not large enough, and all the included articles compared BPPV patients with healthy controls using different parameters of cVEMP. Secondly, all included articles were case-control studies and lack of randomized control trials. Thirdly, the different criteria for defining abnormality of every parameters of cVEMP and some studies without age-matched control existed, probably resulting in heterogeneity. In the future, well-designed prospective case-control studies with age-matched controls and uniform criteria of cVEMP testing should be conducted to investigate the saccular dysfunction compared BPPV patients with healthy controls.

\section{CONCLUSION}

In spite of some shortcomings, we have given a credible conclusion that there are several distinctive characteristics of cVEMP testing in the BPPV patients compared with healthy controls, including longer latency of p13, lower amplitude of p13-n23, and higher proportion of absent response. It is inferred that abnormality of cVEMP may be associated with BPPV occurrence, and neural degeneration in the saccular macula may be a potential pathogenesis for BPPV. 


\section{AUTHOR CONTRIBUTIONS}

GC and GY contributed to the study design, statistical analysis, and manuscript draft. All authors helped to perform the analysis and to revise the manuscript with constructive discussions.

\section{REFERENCES}

1. Colebatch JG, Halmagyi GM, Skuse NF. Myogenic potentials generated by a click-evoked vestibulocollic reflex. J Neurol Neurosurg Psychiatry. (1994) 57:190-7. doi: 10.1136/jnnp.57.2.190

2. Sheykholeslami K, Murofushi T, Kermany MH, Kaga K. Bone-conducted evoked myogenic potentials from the sternocleidomastoid muscle. Acta Oto Laryngol. (2000) 120:731-4. doi: 10.1080/000164800750000252

3. Watson SR, Colebatch JG. Vestibulocollic reflexes evoked by short-duration galvanic stimulation in man. J Physiol. (1998) 513:587-97. doi: 10.1111/j.1469-7793.1998.587bb.x

4. Colebatch JG, Halmagyi GM. Vestibular evoked potentials in human neck muscles before and after unilateral vestibular deafferentation. Neurology. (1992) 42:1635-6. doi: 10.1212/WNL.42.8.1635

5. Curthoys IS, Vulovic V, Burgess AM, Sokolic L, Goonetilleke SC. The response of guinea pig primary utricular and saccular irregular neurons to boneconducted vibration (BCV) and air-conducted sound (ACS). Hear Res. (2016) 331:131-43. doi: 10.1016/j.heares.2015.10.019

6. Rosengren SM, McAngus Todd NP, Colebatch JG. Vestibular-evoked extraocular potentials produced by stimulation with bone-conducted sound. Clin Neurophysiol. (2005) 116:1938-48. doi: 10.1016/j.clinph.2005.03.019

7. Govender S, Dennis DL, Colebatch JG. Vestibular evoked myogenic potentials (VEMPs) evoked by air- and bone-conducted stimuli in vestibular neuritis. Clin Neurophysiol. (2015) 126:2004-13. doi: 10.1016/j.clinph.2014.12.029

8. Rosengren SM, Colebatch JG. The contributions of vestibular evoked myogenic potentials and acoustic vestibular stimulation to our understanding of the vestibular system. Front Neurol. (2018) 9:481. doi: 10.3389/fneur.2018.00481

9. von Brevern M, Radtke A, Lezius F, Feldmann M, Ziese T, Lempert $\mathrm{T}$, et al. Epidemiology of benign paroxysmal positional vertigo: a population based study. J Neurol Neurosurg Psychiatry. (2007) 78:7105. doi: 10.1136/jnnp.2006.100420

10. Akkuzu G, Akkuzu B, Ozluoglu LN. Vestibular evoked myogenic potentials in benign paroxysmal positional vertigo and Meniere's disease. Eur Arch Oto Rhino Laryngol. (2006) 263:510-7. doi: 10.1007/s00405-005-0002-x

11. Parnes LS, McClure JA. Free-floating endolymph particles: a new operative finding during posterior semicircular canal occlusion. Laryngoscope. (1992) 102:988-92. doi: 10.1288/00005537-199209000-00006

12. Lee JD, Park MK, Lee BD, Lee TK, Sung KB, Park JY. Abnormality of cervical vestibular-evoked myogenic potentials and ocular vestibular-evoked myogenic potentials in patients with recurrent benign paroxysmal postitional vertigo. Acta Oto Laryngol. (2013) 133:150-3. doi: 10.3109/00016489.2012.723823

13. Kim EJ, Oh SY, Kim JS, Yang TH, Yang SY. Persistent otolith dysfunction even after successful repositioning in benign paroxysmal positional vertigo. J Neurol Sci. (2015) 358:287-93. doi: 10.1016/j.jns.2015.09.012

14. Korres S, Gkoritsa E, Giannakakou-Razelou D, Yiotakis I, Riga M, Nikolpoulos TP. Vestibular evoked myogenic potentials in patients with BPPV. Med Sci Monit. (2011) 17:Cr42-7. doi: 10.12659/MSM.881328

15. Yang WS, Kim SH, Lee JD, Lee WS. Clinical significance of vestibular evoked myogenic potentials in benign paroxysmal positional vertigo. Otol Neurotol. (2008) 29:1162-6. doi: 10.1097/MAO.0b013e31818a0881

16. Papathanasiou ES, Murofushi T, Akin FW, Colebatch JG. International guidelines for the clinical application of cervical vestibular evoked myogenic potentials: an expert consensus report. Clin Neurophysiol. (2014) 125:65866. doi: 10.1016/j.clinph.2013.11.042

17. Stang A. Critical evaluation of the Newcastle-Ottawa scale for the assessment of the quality of nonrandomized studies in meta-analyses.

\section{SUPPLEMENTARY MATERIAL}

The Supplementary Material for this article can be found online at: https://www.frontiersin.org/articles/10.3389/fneur. 2019.01043/full\#supplementary-material
Eur J Epidemiol. (2010) 25:603-5 doi: 10.1007/s10654-0109491-z

18. Su HC, Huang TW, Young YH, Cheng PW. Aging effect on vestibular evoked myogenic potential. Otol Neurotol. (2004) 25:977-80. doi: $\quad 10.1097 / 00129492-200411000$ 00019

19. Singh NK, Apeksha K. Efficacy of cervical and ocular vestibular-evoked myogenic potentials in evaluation of benign paroxysmal positional vertigo of posterior semicircular canal. Eur Arch Oto Rhino Laryngol. (2016) 273:252332. doi: 10.1007/s00405-015-3867-3

20. Martínez Pascual P, Amaro Merino P. Otolithic damage study in patients with benign paroxysmal positional vertigo with vestibular evoked myogenic potentials. Acta Otorrinolaringol Esp. (2018) 70:131-5. doi: 10.1016/j.otoeng.2018.04.002

21. Xu H, Liang FY, Chen L, Song XC, Tong MC, Thong JF, et al. Evaluation of the utricular and saccular function using oVEMPs and cVEMPs in BPPV patients. J Otolaryngol. (2016) 45:12. doi: 10.1186/s40463-0160125-7

22. Karatas A, Yuce T, Cebi IT, Acar Yuceant G, Haci C, Salviz M Evaluation of cervical vestibular-evoked myogenic potential findings in benign paroxysmal positional vertigo. J Int Adv Otol. (2016) 12:31620. doi: 10.5152/iao.2016.2170

23. Talaat HS, Metwaly MA, Khafagy AH, Abdelraouf HR, Moussa Isak HA. Vestibular evoked myogenic potentials in idiopathic posterior canal benign paroxysmal positional vertigo. Hear Bal Commun. (2013) 11:17681. doi: 10.3109/21695717.2013.834577

24. Nakahara H, Yoshimura E, Tsuda Y, Murofushi T. Damaged utricular function clarified by oVEMP in patients with benign paroxysmal positional vertigo. Acta Oto Laryngol. (2013) 133:144-9. doi: 10.3109/00016489.2012. 720030

25. Longo G, Onofri M, Pellicciari T, Quaranta N. Benign paroxysmal positional vertigo: is vestibular evoked myogenic potential testing useful? Acta Oto Laryngol. (2012) 132:39-43. doi: 10.3109/00016489.2011.619570

26. Eryaman E, Oz ID, Ozker BY, Erbek S, Erbek SS. Evaluation of vestibular evoked myogenic potentials during benign paroxysmal positional vertigo attacks; neuroepithelial degeneration? B-ENT. (2012) 8:247-50.

27. Hall SF, Ruby RR, McClure JA. The mechanics of benign paroxysmal vertigo J Otolaryngol. (1979) 8:151-8.

28. Schuknecht HF. Cupulolithiasis. Arch Otolaryngol. (1969) 90:765-78. doi: 10.1001/archotol.1969.00770030767020

29. Pisani V, Mazzone S, Di Mauro R, Giacomini PG, Di Girolamo S. A survey of the nature of trauma of post-traumatic benign paroxysmal positional vertigo. Int J Audiol. (2015) 54:329-33. doi: 10.3109/14992027.2014.989454

30. Hong SM, Yeo SG. Clinical analysis of patients with idiopathic sudden sensorineural hearing loss and benign paroxysmal positional vertigo. Acta Oto Laryngol. (2013) 133:439-42. doi: 10.3109/00016489.2012.754996

31. Taura A, Funabiki K, Ohgita H, Ogino E, Torii H, Matsunaga M, et al. Onethird of vertiginous episodes during the follow-up period are caused by benign paroxysmal positional vertigo in patients with Meniere's disease. Acta Oto Laryngol. (2014) 134:1140-5. doi: 10.3109/00016489.2014.936624

32. Jang YS, Hwang CH, Shin JY, Bae WY, Kim LS. Age-related changes on the morphology of the otoconia. Laryngoscope. (2006) 116:9961001. doi: $10.1097 / 01 . \mathrm{mlg} .0000217238 .84401 .03$

33. Jeong SH, Kim JS, Shin JW, Kim S, Lee H, Lee AY, et al. Decreased serum vitamin D in idiopathic benign paroxysmal positional vertigo. J Neurol. (2013) 260:832-8. doi: 10.1007/s00415-012-6712-2

34. Gacek RR. Pathology of benign paroxysmal positional vertigo revisited. Ann Otol Rhinol Laryngol. (2003) 112:574-82. doi: 10.1177/000348940311200702 
35. Wang SJ, Chen CN, Hsieh WS, Young YH. Development of vestibular evoked myogenic potentials in preterm neonates. Audiol Neuro Otol. (2008) 13:14552. doi: $10.1159 / 000112422$

36. Naito R, Murofushi T, Mizutani M, Kaga K. Auditory brainstem responses, electrocochleograms, and cochlear microphonics in the myelin deficient mutant hamster 'bt'. Hear Res. (1999) 136:44-8. doi: 10.1016/S0378-5955(99)00107-0

37. Murofushi T, Shimizu K, Takegoshi H, Cheng PW. Diagnostic value of prolonged latencies in the vestibular evoked myogenic potential. Arch Otolaryngol. (2001) 127:1069-72. doi: 10.1001/archotol.127. 9.1069
Conflict of Interest: The authors declare that the research was conducted in the absence of any commercial or financial relationships that could be construed as a potential conflict of interest.

Copyright (c) 2019 Chen, Yu, Li, Zhao, Dai and Wang. This is an open-access article distributed under the terms of the Creative Commons Attribution License (CC BY). The use, distribution or reproduction in other forums is permitted, provided the original author(s) and the copyright owner(s) are credited and that the original publication in this journal is cited, in accordance with accepted academic practice. No use, distribution or reproduction is permitted which does not comply with these terms. 